\title{
DYNAMIC DIFFERENTIAL OLIGOPOLY GAME WITH HYPERBOLIC DEMAND, CUBIC COST AND CAPITAL ACCUMULATION
}

\author{
M. EL AALLAOUI ${ }^{1, *}$, A. KADDAR ${ }^{2}$, A. GOURCH ${ }^{3}$ \\ ${ }^{1}$ Department of Statistics and Applied Mathematics in Economics and Management \\ FSJES Aïn Sebaâ, Hassan II University, Casablanca Morocco. \\ ${ }^{2}$ Department of Economics, FSJES Mohammed V University - Salé Morocco \\ ${ }^{3}$ Department of Economics, FSJES Aïn Sebaâ, Hassan II University, Morocco.
}

*Corresponding author: m.elaallaoui@gmail.com

Received Mar. 7, 2020

\begin{abstract}
Aвstract. A dynamic Cournot oligopoly game, with hyperbolic demand and cubic cost, is proposed. The main aim of this work is to consider the aforementioned problem under a dynamical capital stock constraint (Ramsey's model). We first investigate the existence and uniqueness of optimal solution. Second we prove that the proposed model has two equilibria (Ramsey's and Nash equilibria). Finally, we study the stability of these equilibrium points and their topological classifications. Phase diagrams are established to illustrate our analytical results.
\end{abstract}

2010 Mathematics Subject Classification. 49N90, 49N70.

Key words and phrases. oligopoly; hyperbolic demand; cubic cost; Ramsey growth model; optimal solution; equilibria; stability.

\section{InTRODUCTION}

Interactions between companies can be conducted through quantities (competition in quantities) or prices (price competition). These companies may also try to cooperate to deal with monopoly status. In general, oligopolistic models allow companies to evaluate their strategies theoretically before they are implemented in order to achieve their goals [1-3]. In particular, in [4] Cournot's oligopolistic models are often used to increase the profits of companies competing in price and / or quantity as much as possible. In these models, companies compete

DOI: $10.28924 /$ APJM/7-10 
with their production volumes. In this case, each company, at the same time independently of its competitors, produces its own quantity. This quantity is achieved by knowing the structure of the market (number of competitors) and the demand function, taking into account the dynamical capital stock constraint (Ramsey's model).

The existing literature in this field designs an ideal model for capital stock as a status variable and quantities as control variables, we may cite, for instance, the models [6-8,12]. All the methods mentioned above, propose models with linear or semilinear demand and cost curves, in addition to simplifying calculations and ease of treatment, also ensures existence of optimal solution and the equilibrium, which, in general, is not obvious in the presence of non-linear demand and cost curves [16]. In Industrial Organization, researchers mention the opportunity of dealing with non-linear demand and cost curves. For instance in oligopoly market, the demand for a commodity becomes unitary elastic when the percentage change in the quantity demanded is equal to the percentage change in price. In this case, the numerical value of unitary elastic demand is exactly one and the demand curve is a hyperbolic function $[17,18]$. On the other hand, $[13,15]$ explain that declining average cost throughout the relevant range of outputs is an indicator that the cost function is subadditive. What this requires, however, is that the marginal cost also declines throughout a subset of this range of outputs. And necessary for this is a twice-differentiable cost function, which yields the appropriately shaped average and marginal cost curves. A cubic function has a very flexible specification for modelling total cost, what it is allowed to yield the appropriately shaped average and marginal cost curves $[9,14]$. In this paper, we propose a dynamic oligopoly model with hyperbolic demand, cubic cost and Ramsey capital stock accumulation. and we give sufficient conditions to guarantee that the proposed model has a unique optimal solution, which produces multiple equilibrium points: the Cournot equilibrium of the static configuration and the Ramsey Golden Rule.

The rest of the paper is structured as follows. Section 2 introduces the basics of the model. The open-loop solution is laid out in section 3. Section 4 contains the equilibria analysis: Ramsey equilibrium and Cournot-Nash equilibrium. Section 5 presents the stability analysis and finally we give some concluding remarks.

\section{The SETUP}

Consider an industrial structure in which $N$ identical firms produce and sell individual quantity $q_{i}(t) \in U_{i}=\left\{u \in \mathbb{R}, 0 \leq u \leq u_{\max }(i)\right\}, i=1, \ldots, N$, under Cournot competition. The good is homogeneous, the inverse market demand function for the good is defined by 
hyperbolic demand

$$
p(t)=\frac{A}{Q(t)}, \quad \text { where } \quad A \in \mathbb{R}^{*+} \quad \text { and } \quad Q(t)=\sum_{i=1}^{N} q_{i}(t) .
$$

The total production cost for each firm $i$ for $i=1, \ldots, N$ is given by a cubic function

$$
C\left(q_{i}(t)\right)=a q_{i}^{3}(t)-b q_{i}^{2}(t)+c q_{i}(t)+d,
$$

with $a>0, b>0, c>0, d>0$ and $b^{2} \leq 3 a c$ [9-11]. The total cost function is always increasing, because marginal cost $C^{\prime}\left(q_{i}(t)\right)=3 a q_{i}^{2}(t)-2 b q_{i}(t)+c \geq 0$, and admits a graph initially concave, then convex, as soon as $q>\frac{b}{3 a}$. The marginal cost and the average variable cost initially decrease, reach their minimum points and its start to increase again.

The firm $i$ must calculate the quantities that maximize its profit for each possible level of production of its competitor $\left(q_{1}, \ldots, q_{i-1}, q_{i+1}, \ldots, q_{N}\right)$, so as to determine in advance the best answer it can give it for each of his strategies. It must also neglect the impact of its own production on these quantities since these quantities will not be observed in advance by its competitor. This firm can achieve its main goal by solving the following problem :

$$
\max _{q_{i}} J_{i}\left(q_{i}\right)=\max _{q_{i}} \int_{0}^{+\infty} e^{-\rho t} g_{i}\left(t, q_{i}(t)\right) d t,
$$

under the dynamic constraint (Ramsey problem) $[5,6]$ :

$$
\dot{k}_{i}(t)=f\left(k_{i}(t)\right)-q_{i}(t)-\delta k_{i}(t),
$$

where $J_{i}$ is the own discounted flow of profit of the firm $i, g_{i}\left(t, q_{i}(t)\right)=p(t) q_{i}(t)-C\left(q_{i}(t)\right)$ is the profit of the firm $i, k_{i}$ is the capital stock of the firm $i, \delta$ is the positive depreciation rate of capital stock, $\rho$ is a positive parameter which represents the constant discount rate (possibly depending on monetary factors such as the expected rate of currency depreciation ) and $f$ is the total output produced by firm $i$ at time $t$, satisfying the hypotheses:

$$
\left(H_{01}\right): f^{\prime}>0, \quad\left(H_{02}\right): f^{\prime \prime}<0 .
$$

Therefore, the objective for each firm $i$ is to choose the individual quantity $q_{i}$ witch maximizes the discounted flow of profits.

$$
J_{i}\left(q_{i}\right)=\int_{0}^{+\infty} e^{-\rho t} g_{i}\left(t, q_{i}(t)\right) d t
$$

where

$$
g_{i}\left(t, q_{i}(t)\right)=p(t) q_{i}(t)-C\left(q_{i}(t)\right)=\frac{A q_{i}(t)}{Q(t)}-a q_{i}^{3}(t)+b q_{i}^{2}(t)-c q_{i}(t)-d .
$$


Since the profit function $g_{i}$ is continuous with respect to quantity $q_{i}$ and $q_{i}(t) \in\left[0, u_{\max }(i)\right]$, the profit $g_{i}$ is bounded at any time $t$, i.e there exists $M>0$, such as

$$
\left|g_{i}\left(t, q_{i}(t)\right)\right| \leq M, \quad \forall t \in[0,+\infty)
$$

Then the improper integral in (2.5) might converge for any quantity $q_{i}$,

$$
J_{i}\left(q_{i}\right)=\lim _{T \rightarrow+\infty} J_{i}\left(T, q_{i}\right)=\lim _{T \rightarrow+\infty} \int_{0}^{T} e^{-\rho t} g_{i}\left(t, q_{i}(t)\right) d t \leq \lim _{T \rightarrow+\infty} \int_{0}^{T} M e^{-\rho t} .
$$

\section{THE OPEN-LOOP SOLUTION}

The optimal control problem associated is given by

$$
\max _{q_{i}} J_{i}\left(q_{i}\right)=\max _{q_{i}} \int_{0}^{+\infty} e^{-\rho t} g_{i}\left(t, q_{i}(t)\right) d t
$$

under the dynamic constraints

$$
\dot{k}(t)=\mathbf{f}(k(t))-q(t)-\delta k(t)
$$

where $k(t)=\left(\begin{array}{llllll}k_{1}(t) & \ldots & k_{i}(t) & \ldots & k_{N}(t)\end{array}\right)^{\mathrm{T}}$ and $q(t)=\left(\begin{array}{lllll}q_{1}(t) & \ldots & q_{i}(t) & \ldots & q_{N}(t)\end{array}\right)^{\mathrm{T}}$ are the vectors of states and controls, $\left.\mathbf{f}(k(t))=\left(\begin{array}{lllll}f\left(k_{1}(t)\right) & \ldots & f\left(k_{i}(t)\right.\end{array}\right) \quad \ldots \quad f\left(k_{N}(t)\right)\right)^{\mathrm{T}}$, with initial condition

$$
k(0)=\left(\begin{array}{lllll}
k_{01} & \ldots & k_{0 i} & \ldots & k_{0 N}
\end{array}\right)^{\mathrm{T}} .
$$

For any control $\bar{q}(t)$, the above regularity assumptions of the function $f$ guarantee that a unique solution $\bar{k}$ exists of the Cauchy problem (3.2), the pair $(\bar{k}, \bar{q})$ is called admissible pair for the state equation, and satisfies the corresponding integral equation

$$
\bar{k}_{i}(t)=k_{0 i}+\int_{0}^{t} f\left(\bar{k}_{i}(s)\right)-\bar{q}_{i}(s)-\delta \bar{k}_{i}(s) d s .
$$

The current value Hamiltonian for each firm $i$ is

$$
H_{i}\left(t, k, q_{i}, \lambda_{i}\right)=e^{-\rho t} g_{i}\left(t, q_{i}(t)\right)+\left\langle\lambda_{i} \mid \mathbf{f}(k(t))-q(t)-\delta k(t)\right\rangle,
$$

where $\lambda_{i}(t)=\left(\begin{array}{lllll}\lambda_{i 1}(t) & \ldots & \lambda_{i i}(t) & \ldots & \lambda_{i N}(t)\end{array}\right)^{\mathrm{T}}$ is the costate variables associated with states by firm $i$, then

$$
H_{i}\left(t, k, q, \lambda_{i}\right)=e^{-\rho t} g_{i}\left(t, q_{i}(t)\right)+\sum_{j=1}^{N} \lambda_{i j}\left(f\left(k_{j}(t)\right)-q_{j}(t)-\delta k_{j}(t)\right) .
$$

The necessary conditions in order that $\left(q, k, \lambda_{i}\right)$ to be optimal for (3.1) and (3.2), are:

$$
\frac{\partial H_{i}}{\partial q_{i}}\left(t, q, k, \lambda_{i}\right)=e^{-\rho t} \frac{\partial g_{i}}{\partial q_{i}}\left(t, q_{i}\right)-\lambda_{i i}(t)=0,
$$




$$
\begin{gathered}
\frac{\partial H_{i}}{\partial k_{i}}\left(t, q, k, \lambda_{i}\right)=\lambda_{i i}(t)\left(f^{\prime}\left(k_{i}(t)\right)-\delta\right)=-\dot{\lambda}_{i i}(t), \\
\frac{\partial H_{i}}{\partial k_{j}}\left(t, q, k, \lambda_{i}\right)=\lambda_{i j}(t)\left(f^{\prime}\left(k_{j}(t)\right)-\delta\right)=-\dot{\lambda}_{i j}(t) \quad \forall j \neq i .
\end{gathered}
$$

The following theorem gives a sufficient conditions to guarantee that the dynamic oligopoly model proposed admits a unique optimal solution.

Theorem 3.1. Let $\left(q_{i}^{*}, k_{i}^{*}\right)$ be an admissible pair for the state equation 2.4. Suppose that $\left(q_{i}^{*}, k_{i}^{*}\right)$ satisfy the necessary conditions (3.7) and (3.8) with costate vector $\lambda_{i i}^{*}$. If :

$$
\frac{\partial g_{i}}{\partial q_{i}}\left(t, q_{i}^{*}\right) \geq 0, \quad \forall t \in[0,+\infty)
$$

$$
\lim _{t \longrightarrow+\infty} \frac{\partial g_{i}}{\partial q_{i}}\left(t, q_{i}^{*}\right) k_{i}^{*}(t)=0,
$$

$$
\frac{\partial^{2} g_{i}}{\partial^{2} q_{i}}\left(t, q_{i}\right)<0, \quad \forall q_{i} .
$$

Then for every admissible pair $\left(\bar{k}_{i}, \bar{q}_{i}\right)$ for the state equation 2.4

$$
J_{i}\left(\bar{q}_{i}\right)<J_{i}\left(q_{i}^{*}\right) .
$$

Proof. The condition (3.12) proves that for any admissible pair $\left(\bar{k}_{i}, \bar{q}_{i}\right)$,

$$
g_{i}\left(t, \bar{q}_{i}\right)-g_{i}\left(t, q_{i}^{*}\right)<\frac{\partial g_{i}}{\partial q_{i}}\left(t, q_{i}^{*}\right)\left(\bar{q}_{i}-q_{i}^{*}\right) .
$$

The necessary condition (3.7) implies that:

$$
e^{-\rho t} g_{i}\left(\bar{q}_{i}\right)-e^{-\rho t} g_{i}\left(q_{i}^{*}\right)<\lambda_{i i}^{*}\left(\bar{q}_{i}-q_{i}^{*}\right),
$$

then, for each $T>0$ we have

$$
J_{i}(T, \bar{q})-J_{i}\left(T, q^{*}\right)=\int_{0}^{T} e^{-\rho t} g_{i}\left(\bar{q}_{i}\right)-e^{-\rho t} g_{i}\left(q_{i}^{*}\right) d t<\int_{0}^{T} \lambda_{i i}^{*}\left(\bar{q}_{i}-q_{i}^{*}\right) d t .
$$

The necessary condition (3.8) implies that

$$
\begin{aligned}
\lambda_{i i}^{*}\left(\bar{q}_{i}-q_{i}^{*}\right) & =\lambda_{i i}^{*}\left(\bar{q}_{i}-q_{i}^{*}\right)-\left(\lambda_{i i}^{*} f^{\prime}\left(k_{i}^{*}\right)-\lambda_{i i}^{*} \delta+\dot{\lambda}_{i i}^{*}\right)\left(\bar{k}_{i}-k_{i}^{*}\right) \\
& =\lambda_{i i}^{*}\left(\bar{q}_{i}+\delta \bar{k}_{i}-q_{i}^{*}-\delta k_{i}^{*}\right)-\dot{\lambda}_{i i}^{*}\left(\bar{k}_{i}-k_{i}^{*}\right)-\lambda_{i i}^{*} f^{\prime}\left(k_{i}^{*}\right)\left(\bar{k}_{i}-k_{i}^{*}\right) .
\end{aligned}
$$


The pairs $(\bar{k}, \bar{q})$ and $\left(q^{*}, k^{*}\right)$ are two admissible pairs, thus

$$
\begin{aligned}
\lambda_{i i}^{*}\left(\bar{q}_{i}-q_{i}^{*}\right) & =\lambda_{i i}^{*}\left(f\left(\bar{k}_{i}\right)-\dot{\bar{k}}_{i}-f\left(k_{i}^{*}\right)+\dot{k}^{*}{ }_{i}\right)-\dot{\lambda}_{i i}^{*}\left(\bar{k}_{i}-k_{i}^{*}\right)-\lambda_{i i}^{*} f^{\prime}\left(k_{i}^{*}\right)\left(\bar{k}_{i}-k_{i}^{*}\right) \\
& =-\dot{\lambda}_{i i}^{*}\left(\bar{k}_{i}-k_{i}^{*}\right)-\lambda_{i i}^{*}\left(\dot{\bar{k}}_{i}-\dot{k}_{i}^{*}\right)+\lambda_{i i}^{*}\left(f\left(\bar{k}_{i}\right)-f\left(k_{i}^{*}\right)-f^{\prime}\left(k_{i}^{*}\right)\left(\bar{k}_{i}-k_{i}^{*}\right)\right) .
\end{aligned}
$$

The function $f$ is concave so the expression $f\left(\bar{k}_{i}\right)-f\left(k_{i}^{*}\right)-f^{\prime}\left(k_{i}^{*}\right)\left(\bar{k}_{i}-k_{i}^{*}\right)$ is negative. Hence, noting that $\lambda_{i i}^{*}(t)=e^{-\rho t} \frac{\partial g_{i}}{\partial q_{i}}\left(t, q_{i}^{*}\right) \geq 0$ by assumption, we have

$$
\lambda_{i i}^{*}\left(\bar{q}_{i}-q_{i}^{*}\right) \leq-\dot{\lambda}_{i i}^{*}\left(\bar{k}_{i}-k_{i}^{*}\right)-\lambda_{i i}^{*}\left(\dot{\bar{k}}_{i}-\dot{k}_{i}^{*}\right) .
$$

The equalities (3.16) and (3.17) imply that

$$
J_{i}(T, \bar{q})-J_{i}\left(T, q^{*}\right)<-\int_{0}^{T} \dot{\lambda}_{i i}^{*}\left(\bar{k}_{i}-k_{i}^{*}\right)+\lambda_{i i}^{*}\left(\dot{\bar{k}}_{i}-\dot{k}_{i}^{*}\right) d t .
$$

One has

$$
\begin{aligned}
\int_{0}^{T} \dot{\lambda}_{i i}^{*}\left(\bar{k}_{i}-k_{i}^{*}\right)+\lambda_{i i}^{*}\left(\dot{\bar{k}}_{i}-\dot{k}_{i}^{*}\right) d t & \left.=\left[\lambda_{i i}^{*}(t)\left(\bar{k}_{i}(t)-k_{i}^{*}\right)(t)\right)\right]_{0}^{T} \\
& =\lambda_{i i}^{*}(T)\left(\bar{k}_{i}(T)-k_{i}^{*}(T)\right)-\lambda_{i i}^{*}(0)\left(\bar{k}_{i}(0)-k_{i}^{*}(0)\right)
\end{aligned}
$$

and by admissibility we have $k_{i}^{*}(0)=\bar{k}_{i}(0)=k_{0 i}$. Hence

$$
\int_{0}^{T} \dot{\lambda}_{i i}^{*}\left(\bar{k}_{i}-k_{i}^{*}\right)+\lambda_{i i}^{*}\left(\dot{\bar{k}}_{i}-{\dot{k^{*}}}_{i}\right)=\lambda_{i i}^{*}(T)\left(\bar{k}_{i}(T)-k_{i}^{*}(T)\right),
$$

and

$$
J_{i}(T, \bar{q})-J_{i}\left(T, q^{*}\right)<-\lambda_{i i}^{*}(T)\left(\bar{k}_{i}(T)-k_{i}^{*}(T)\right)
$$

Since $\lambda_{i i}^{*}=\frac{\partial g_{i}}{\partial q_{i}}\left(t, q_{i}^{*}\right) \geq 0$, one has

$$
J_{i}\left(T, \bar{q}_{i}\right)-J_{i}\left(T, q_{i}^{*}\right)<\lambda_{i i}^{*}(T) k_{i}^{*}(T)-\lambda_{i i}^{*}(T) \bar{k}_{i}(T) \leq \lambda_{i i}^{*}(T) k_{i}^{*}(T) .
$$

Letting $T \rightarrow+\infty$ yield $J_{i}\left(\bar{q}_{i}\right)-J_{i}\left(q_{i}^{*}\right)<\lim _{t \rightarrow+\infty} e^{-\rho t} \frac{\partial g_{i}}{\partial q_{i}}\left(t, q_{i}^{*}\right) k_{i}^{*}(t)=0$, then it follows that $J_{i}\left(\bar{q}_{i}\right)<J_{i}\left(q_{i}^{*}\right)$ for all admissible control path $\bar{q}_{i}$.

Condition (3.11) is a transversality condition; we can better understand the meaning of this condition if we consider our problem with a finite horizon $t \in[0, T]$. The function $g_{i}$ is concave, and reaches its maximum at $q_{i}^{\max }$ solution of $\frac{\partial g_{i}}{\partial q_{i}}\left(t, q_{i}\right)=0$. Hence, if $\lambda_{i i}^{*}(T)=e^{-\rho T} \frac{\partial g_{i}}{\partial q_{i}}\left(T, q_{i}^{*}\right)>0$, then $q_{i}^{*}(T)<q^{\max }$, in this cas $k_{i}^{*}(T)=0$, because if the capital $k_{i}^{*}(T)>0$, we can affect the payoff $g_{i}$ by consuming this capital. Therefore, we have tree cases:

Case I:: $\lambda_{i i}^{*}(T)=e^{-\rho T} \frac{\partial g_{i}}{\partial q_{i}}\left(T, q_{i}^{*}\right)>0$ and $k_{i}^{*}(T)=0$.

Case II:: $\lambda_{i i}^{*}(T)=e^{-\rho T} \frac{\partial g_{i}}{\partial q_{i}}\left(T, q_{i}^{*}\right)=0$ and $k_{i}^{*}(T)>0$. 
Case III:: $\lambda_{i i}^{*}(T)=e^{-\rho T} \frac{\partial g_{i}}{\partial q_{i}}\left(T, q_{i}^{*}\right)=0$ and $k_{i}^{*}(T)=0$.

Hence, $\lambda_{i i}^{*}(T) k_{i}^{*}(T)=0$. The transversality condition can be seen as the limit of this condition when $T \rightarrow+\infty$.

\section{EQUILIBRIUM ANALYSIS}

In this section we establish the state-control dynamic system of the open-loop solution, and we use this system to determine the equilibrium points. for each $t>0$ we have

$$
g_{i}\left(t, q_{i}(t)\right)=p(Q(t)) q_{i}(t)-C\left(q_{i}(t)\right)=p\left(q_{i}(t)+Q_{-i}(t)\right) q_{i}(t)-C\left(q_{i}(t)\right)
$$

where

$$
Q_{-i}(t)=Q(t)-q_{i}(t)
$$

The first necessary condition (3.7), implies that

$$
\dot{\lambda}_{i i}(t)=-\rho \lambda_{i i}+e^{-\rho t} \frac{\partial^{2} g_{i}}{\partial^{2} q_{i}} \dot{q}+e^{-\rho t} \frac{\partial^{2} g_{i}}{\partial Q_{-i} \partial q_{i}} \dot{Q}_{-i} .
$$

Using the second necessary condition (3.8), the equation (4.3) can be rewritten as follows:

$$
\lambda_{i i}\left(\delta+\rho-f^{\prime}\left(k_{i}\right)\right)=e^{-\rho t} \frac{\partial^{2} g_{i}}{\partial^{2} q_{i}} \dot{q}+e^{-\rho t} \frac{\partial^{2} g_{i}}{\partial Q_{-i} \partial q_{i}} \dot{Q}_{-i}
$$

then, from the first necessary condition (3.7), we have

$$
\frac{\partial g_{i}}{\partial q_{i}}\left(\delta+\rho-f^{\prime}\left(k_{i}\right)\right)=\frac{\partial^{2} g_{i}}{\partial^{2} q_{i}} \dot{q}_{i}+\frac{\partial^{2} g_{i}}{\partial Q_{-i} \partial q_{i}} \dot{Q}_{-i},
$$

with

$$
\begin{gathered}
\frac{\partial g_{i}}{\partial q_{i}}=A\left(q_{i}+Q_{-i}\right)^{-1}-A\left(q_{i}+Q_{-i}\right)^{-2} q_{i}-3 a q_{i}^{2}+2 b q_{i}-c, \\
\frac{\partial^{2} g_{i}}{\partial^{2} q_{i}}=-2 A\left(q_{i}+Q_{-i}\right)^{-2}+2 A\left(q_{i}+Q_{-i}\right)^{-3} q_{i}-6 a q_{i}+2 b, \\
\frac{\partial^{2} g_{i}}{\partial Q_{-i} \partial q_{i}}=-A\left(q_{i}+Q_{-i}\right)^{-2}+2 A\left(q_{i}+Q_{-i}\right)^{-3} q_{i},
\end{gathered}
$$

Substituting the above expressions in (4.5), one obtains:

$$
\begin{aligned}
& \left(A\left(q_{i}+Q_{-i}\right)^{-1}-A\left(q_{i}+Q_{-i}\right)^{-2} q_{i}-3 a q_{i}^{2}+2 b q_{i}-c\right)\left(\delta+\rho-f^{\prime}\left(k_{i}\right)\right)= \\
& \left(-2 A\left(q_{i}+Q_{-i}\right)^{-2}+2 A\left(q_{i}+Q_{-i}\right)^{-3} q_{i}-6 a q_{i}+2 b\right) \dot{q}_{i} \\
& +\left(-A\left(q_{i}+Q_{-i}\right)^{-2}+2 A\left(q_{i}+Q_{-i}\right)^{-3} q_{i}\right) \dot{Q}_{-i} .
\end{aligned}
$$


Given the ex-ante symmetry, we impose that the choices made by the firms are symmetrical, i.e.:

$$
q_{i}=q_{j}=q \quad \forall i \neq j
$$

Under the above assumption, we obtain

$$
\dot{q}\left(\tilde{N} A q^{-2}+6 a q-2 b\right)=l(q)\left(\delta+\rho-f^{\prime}(k)\right),
$$

where

$$
l(q)=3 a q^{2}-2 b q+c-\tilde{N} A q^{-1}
$$

and

$$
\tilde{N}=\frac{N-1}{N^{2}}
$$

The condition (3.12) and the assumption (4.9), imply that

$$
\tilde{N} A q^{-2}+6 a q-2 b>\frac{2 \tilde{N}}{N} A q^{-2}+6 a q-2 b=-\frac{\partial^{2} g_{i}}{\partial^{2} q}(t, q)>0 .
$$

Hence, the control dynamic equation is

$$
\dot{q}=h(q)\left(\delta+\rho-f^{\prime}(k)\right),
$$

where

$$
h(q)=\frac{l(q)}{\tilde{N} A q^{-2}+6 a q-2 b}=\frac{3 a q^{2}-2 b q+c-\tilde{N} A q^{-1}}{\tilde{N} A q^{-2}+6 a q-2 b} .
$$

The state-control dynamic system of the model is the following one:

$$
\left\{\begin{array}{l}
\dot{k}=f(k)-q-\delta k \\
\dot{q}=h(q)\left(\delta+\rho-f^{\prime}(k)\right) .
\end{array}\right.
$$

Imposing stationarity on (4.16), we see that

$$
\dot{q}=0 \Longleftrightarrow\left\{\begin{array}{l}
\delta+\rho-f^{\prime}(k)=0, \\
l(q)=3 a q^{2}-2 b q+c-\tilde{N} A q^{-1}=0 .
\end{array}\right.
$$

The first equation characterizes the Ramsey golden rule, and the second equation characterizes the Cournot-Nash equilibrium of the static version of the Cournot game with the same demand and cost functions

$$
\max _{q_{i}} g_{i}\left(t, q_{i}\right)
$$


4.1. Ramsey golden rule equilibrium. Since the function $f$ is strictly concave, there is only one value of $k$ such that the $f^{\prime}(k)$ is equal to $(\delta+\rho)$. this value is the symmetric closed-loop Ramsey golden rule solution $k_{R}$, the corresponding quantity is $q_{R}=f\left(k_{R}\right)-\delta k_{R}$. Since $f$ is concave, decreasing $k$ will raise $f^{\prime}(k)$. therefore, we must have

$$
f^{\prime}(k)-\delta-\rho>0, \quad \forall k<k_{R}
$$

$$
f^{\prime}(k)-\delta-\rho=0, \quad k=k_{R}
$$

4.2. Cournot-Nash equilibrium. The following theorem prove that the open-loop solution admits a unique symmetric Cournot-Nash equilibrium.

Theorem 4.1. The differential oligopoly game with capital accumulation (3.1) and (3.2) admits a unique symmetric closed-loop Cournot-Nash equilibrium:

$$
q_{C N}=\sqrt[3]{\frac{-\mu+\sqrt{\Delta}}{2}}+\sqrt[3]{\frac{-\mu-\sqrt{\Delta}}{2}}+\frac{2 b}{3^{2} a},
$$

where

$$
\begin{gathered}
\Delta=\mu^{2}+\frac{2^{2}}{3^{3}} \beta^{3}>0, \\
\mu=\frac{-2^{4} b^{3}-3^{5} \tilde{N} a^{2} A+3^{3} 2 a b c}{3^{6} a^{3}} ; \quad \tilde{N}=\frac{N-1}{N^{2}}, \\
\beta=\frac{3^{2} a c-2^{2} b^{2}}{3^{3} a^{2}} .
\end{gathered}
$$

Proof. The Cournot-Nash equilibrium is given by the solution of equation $l(q)=0$. One has

$$
l(q)=3 a q^{2}-2 b q+c-\tilde{N} A q^{-1}=0 \Longleftrightarrow \tilde{l}(q)=3 a q^{3}-2 b q^{2}+c q-\tilde{N} A=0 .
$$

If $9 a c<4 b^{2}$, the quadratic equation $\tilde{l}^{\prime}(q)=9 a q^{2}-4 b q+c=0$ has two strictly positive roots $q_{1}=\frac{2 b-\sqrt{4 b^{2}-9 a c}}{9 a}>0$ and $q_{2}=\frac{2 b+\sqrt{4 b^{2}-9 a c}}{9 a}>0$. Hence, the function $\tilde{l}^{\prime}(q)$ is strictly positive in ]$-\infty, q_{1}[\cup] q_{2},+\infty[$, and the property (4.13) implies that

$$
\tilde{l}\left(q_{1}\right)=\tilde{l}\left(q_{1}\right)+\tilde{l}^{\prime}\left(q_{1}\right)=-6 a q_{1}^{2}+2 b q_{1}-\tilde{N} A<0,
$$


and

$$
\tilde{l}\left(q_{2}\right)=\tilde{l}\left(q_{2}\right)+\tilde{l}^{\prime}\left(q_{2}\right)=-6 a q_{2}^{2}+2 b q_{2}-\tilde{N} A<0 .
$$

If $9 a c \geq 4 b^{2}$ the function $\tilde{l}^{\prime}(q)$ is strictly positive in ] $-\infty+\infty[$.

As $\lim _{q \rightarrow-\infty} \tilde{l}(0)=-\infty, \tilde{l}\left(q_{1}\right)<0, \tilde{l}\left(q_{2}\right)<0$ and $\tilde{l}$ strictly increases to $+\infty$ when $q \longrightarrow+\infty$, the cubic equation $\tilde{l}(q)=0$ admits a unique real root $q_{C N}>0$.

The change of variable $y=q-\frac{2 b}{3^{2} a}$, transforms the equation $\tilde{l}(q)=0$ to reduced form

$$
y^{3}+\beta y+\mu=0,
$$

where

$$
\beta=\frac{3^{2} a c-2^{2} b^{2}}{3^{3} a^{2}} \quad \text { and } \quad \mu=\frac{-2^{4} b^{3}-3^{5} \tilde{N} a^{2} A+3^{3} 2 a b c}{3^{6} a^{3}} .
$$

The algebraic solution of the cubic equation (4.26) giving by Cardano's Method. The numbers of real and complex roots are determined by the discriminant of the cubic equation

$$
\Delta=\mu^{2}+\frac{2^{2}}{3^{3}} \beta^{3} .
$$

Since the cubic equation $\tilde{l}(q)=0$ has unique real root, the cubic equation(4.26) has unique real root, two non-real complex conjugate roots and the discriminant $\Delta>0$. We pose

$$
u=\sqrt[3]{\frac{-\mu+\sqrt{\Delta}}{2}} \text { and } v=\sqrt[3]{\frac{-\mu-\sqrt{\Delta}}{2}} .
$$

The real root is $y_{0}=u+v$, and the two non-real complex conjugate roots are:

$$
\left\{\begin{array}{l}
y_{1}=j u+\bar{j} v, \\
y_{2}=j^{2} u+\overline{j^{2}} v .
\end{array} \text { where } j=e^{i \frac{2 \pi}{3}} \text { and } j^{2}=e^{i \frac{4 \pi}{3}} .\right.
$$

Hence,

$$
q_{C N}=y_{0}+\frac{2 b}{3^{2} a}=u+v+\frac{2 b}{3^{2} a} .
$$

Notice that for the symmetric closed-loop Cournot-Nash equilibrium $q_{C N}$, the corresponding capital stock given by solving the equation

$$
q_{C N}=F\left(k_{C N}\right),
$$

with

$$
F(k)=f(k)-\delta k .
$$


Since $F$ is strictly concave and reaches its maximum in $k=k_{o r}$ solution of $f^{\prime}\left(k_{o r}\right)=\delta$, if $q_{C N}<F\left(k_{o r}\right)$ there exist two corresponding capital stocks $k_{C N 1}<k_{o r}$ and $k_{C N 2}>k_{o r}$, and unique corresponding capital stock $k_{C N}=k_{o r}$ if $q_{C N}=F\left(k_{o r}\right)$. As $f$ is strictly concave, the equations $f^{\prime}\left(k_{o r}\right)=\delta$ and $f^{\prime}\left(k_{R}\right)=\rho+\delta$ imply that $k_{R}<k_{o r}$.

The graph $C_{l}$ of the polynomial $\tilde{l}(q)=3 a q^{3}-2 b q^{2}+c q-\tilde{N} A$ starts by $-\tilde{N} A$, moves steeply upward, crosses the $q$-axis at the root $q_{C N}$, and then moves steeply upward again. Changing the coefficient $-\tilde{N} A$ move $C_{l}$ vertically-up or down, and Changing $a, b, c$ alter the curvature of the parabolic element. Therefore the symmetric closed-loop Cournot-Nash equilibrium $q_{C N}=0$ if $\tilde{N} A=0$, and increases when $-\tilde{N} A$ shifts downwards and the coefficients $a, b, c$ are increase. We obtain one out of five possible regimes:

(1) Case 1: If $q_{C N}<q_{R}$ we have $k_{C N 1}<k_{R}<k_{o r}<k_{C N 2}$ and there exist three steady state points, with $E_{1}=\left(q_{C N}, k_{C N 1}\right), E_{2}=\left(q_{R}, k_{R}\right)$ and $E_{3}=\left(q_{C N}, k_{C N 2}\right)$.

(2) Case 2: If $q_{C N}=q_{R}$ we have $k_{C N 1}=k_{R}<k_{o r}<k_{C N 2}$ and there exist two steady state points, with $E_{1}=E_{2}=\left(q_{C N}, k_{C N 1}\right)=\left(q_{R}, k_{R}\right)$ and $E_{3}=\left(q_{C N}, k_{C N 2}\right)$.

(3) Case 3: If $q_{R}<q_{C N}<F\left(k_{o r}\right)$ we have $k_{R}<k_{C N 1}<k_{o r}<k_{C N 2}$ and there exist three steady state points, with $E_{1}=\left(q_{C N}, k_{C N 1}\right), E_{2}=\left(q_{R}, k_{R}\right)$ and $E_{3}=\left(q_{C N}, k_{C N 2}\right)$.

(4) Case 4: If $q_{R}<q_{C N}=F\left(k_{o r}\right)$ we have $k_{R}<k_{C N 1}=k_{C N 2}=k_{o r}$ and there exist two steady state points, with $E_{2}=\left(q_{R}, k_{R}\right)$ and $E_{1}=E_{3}=\left(q_{C N}, k_{o r}\right)$.

(5) Case 5: If $q_{R}<F\left(k_{o r}\right)<q_{C N}$ there exists a unique steady state point, $E_{2}=\left(q_{R}, k_{R}\right)$.

Proposition 4.1. For all $q \in(0,+\infty)$, one has:

$$
h(q)=\frac{\left(q-q_{C N}\right)\left(3 a q^{2}+\left(-2 b+3 a q_{C N}\right) q+\tilde{N} A / q_{C N}\right)}{\tilde{N} A q^{-1}+6 a q^{2}-2 b q}
$$

and

$$
3 a q^{2}+\left(-2 b+3 a q_{C N}\right) q+\tilde{N} A / q_{C N}>0
$$

Proof. Since the cubic polynomial $\tilde{l}$ has one real root $q_{C N}$ and two non-real complex conjugate roots, there exist three scalars $\tilde{a}, \tilde{b}$ and $\tilde{c}$ such that $\tilde{l}(q)=\left(q-q_{C N}\right)\left(\tilde{a} q^{2}+\tilde{b} q+\tilde{c}\right)$. As $\left(q-q_{C N}\right)\left(\tilde{a} q^{2}+\tilde{b} q+\tilde{c}\right)=3 a q^{3}-2 b q^{2}+c q-\tilde{N} A$, one has $\tilde{a} q^{3}+\left(\tilde{b}-\tilde{a} q_{C N}\right) x^{2}+\left(\tilde{c}-\tilde{b} q_{C N}\right) q-\tilde{c} q_{C N}=$ 
$3 a q^{3}-2 b q^{2}+c q-\tilde{N} A$, therefore one can identify the values of $\tilde{a}, \tilde{b}$ and $\tilde{c}$

$$
\left\{\begin{array} { l } 
{ \tilde { a } = 3 a } \\
{ \tilde { b } - \tilde { a } q _ { C N } = - 2 b } \\
{ \tilde { c } - \tilde { b } q _ { C N } = c } \\
{ \tilde { c } q _ { C N } = \tilde { N } A }
\end{array} \Longrightarrow \left\{\begin{array}{l}
\tilde{a}=3 a, \\
\tilde{b}=-2 b+3 a q_{C N} \\
\tilde{c}=\tilde{N} A / q_{C N}
\end{array}\right.\right.
$$

Hence,

$$
h(q)=\frac{\tilde{l}(q)}{\tilde{N} A q^{-1}+6 a q^{2}-2 b q}=\frac{\left(q-q_{C N}\right)\left(3 a q^{2}+\left(-2 b+3 a q_{C N}\right) q+\tilde{N} A / q_{C N}\right)}{\tilde{N} A q^{-1}+6 a q^{2}-2 b q} .
$$

The quadratic equation $3 a q^{2}+\left(-2 b+3 a q_{C N}\right) q+\tilde{N} A / q_{C N}=0$ has two non-real complex conjugate roots, therefore the sign of polynomial $3 a q^{2}+\left(-2 b+3 a q_{C N}\right) q+\tilde{N} A / q_{C N}$ is the same as the sign of the coefficient $a$.

Since the reason why the locus $\dot{q}=0$ is $k=k_{R}$ or $q=q_{C N}$, the curve $\dot{q}=0$ is two straight lines given by $k=k_{R}$ and $q=q_{C N}$. The locus $\dot{k}=0$ is given by the solutions of the equation $q=F(k)=f(k)-\delta k$. Therefore the curve $\dot{k}=0$ is strictly concave and reaches its maximum in $k_{\text {or }}$.

The state-control dynamic system (4.16) and proposition 3.1 imply that

- Above the curve $\dot{k}=0 ; \dot{k}<0$ and below this curve $\dot{k}>0$.

- if $k<k_{R}$, Above the straight line $q=q_{C N} ; \dot{q}<0$ and below this straight line $\dot{q}>0$, and the opposite if $k>k_{R}$.

- if $q<q_{C N}$, to the left of the straight line $k=k_{R} ; \dot{q}>0$ and to the right $\dot{q}<0$, and the opposite if $q>q_{C N}$.

\section{StABility}

To assess the stability properties of the steady states, consider the Jacobian matrix associated to (4.16):

$$
J(k, q)=\left(\begin{array}{cc}
\frac{\partial \dot{k}}{\partial k} & \frac{\partial \dot{k}}{\partial q} \\
\frac{\partial \dot{q}}{\partial k} & \frac{\partial \dot{q}}{\partial q}
\end{array}\right)=\left(\begin{array}{cc}
f^{\prime}(k)-\delta & -1 \\
h(q) f^{\prime \prime}(k) & h^{\prime}(q)\left(\delta+\rho-f^{\prime}(k)\right)
\end{array}\right) .
$$

Evaluating $J$ in the Ramsey golden rule Equilibrium yields

$$
J_{R}=J\left(k_{R}, q_{R}\right)=\left(\begin{array}{cc}
\rho & -1 \\
h\left(q_{R}\right) f^{\prime \prime}\left(k_{R}\right) & 0
\end{array}\right) .
$$


Since $J_{R}$ a square matrix of order 2 , its characteristic polynomial $P_{R}(X)=\operatorname{det}\left(J_{R}-X I_{2}\right)$ can write explicitly in terms of its determinant and its trace .

$$
P_{R}(X)=X^{2}-\operatorname{tr}\left(J_{R}\right) X+\operatorname{det}\left(J_{R}\right)
$$

The nature of the two roots $\eta_{1}$ and $\eta_{2}$ is determined by the discriminant $\Delta_{R}=\operatorname{tr}\left(J_{R}\right)^{2}-4 \operatorname{det}\left(J_{R}\right)$ and knowing the determinant and trace of $J_{R}$ allows us to deduce their signs,

$$
\left\{\begin{array}{l}
\eta_{1}+\eta_{2}=\operatorname{tr}\left(J_{R}\right)=\rho \\
\eta_{1} \eta_{2}=\operatorname{det}\left(J_{R}\right)=h\left(q_{R}\right) f^{\prime \prime}\left(k_{R}\right) .
\end{array}\right.
$$

Evaluating $J$ in the Cournot-Nash equilibrium yields

$$
J_{C N i}=J\left(k_{C N i}, q_{C N}\right)=\left(\begin{array}{cc}
f^{\prime}\left(k_{C N i}\right)-\delta & -1 \\
0 & h^{\prime}\left(q_{C N i}\right)\left(\delta+\rho-f^{\prime}\left(k_{C N i}\right)\right)
\end{array}\right) \quad i \in\{1,2\} .
$$

As $h^{\prime}(q)=1-h(q) \frac{6 a-2 \tilde{N} A q^{-3}}{\tilde{N} A q^{-2}+6 a q-2 b}$, the properties $h\left(q_{C N i}\right)=0, f^{\prime}\left(k_{o r}\right)=\delta$ and $f^{\prime}\left(k_{R}\right)=\delta+\rho$ imply that

$$
J_{C N i}=\left(\begin{array}{cc}
f^{\prime}\left(k_{C N i}\right)-f^{\prime}\left(k_{o r}\right) & -1 \\
0 & f^{\prime}\left(k_{R}\right)-f^{\prime}\left(k_{C N i}\right)
\end{array}\right) .
$$

The matrix $J_{C N i}$ has two real eigenvalues $\mu_{1 i}=f^{\prime}\left(k_{C N i}\right)-f^{\prime}\left(k_{o r}\right)$ and $\mu_{2 i}=f^{\prime}\left(k_{R}\right)-f^{\prime}\left(k_{C N i}\right)$.

5.1. Case 1: $q_{C N}<q_{R}$. As $\operatorname{det}\left(J_{R}\right)=h\left(q_{R}\right) f^{\prime \prime}\left(k_{R}\right)<0$, the matrix $J_{R}$ has two real eigenvalues $\eta_{1}=\frac{\rho-\sqrt{\Delta_{R}}}{2}$ and $\eta_{2}=\frac{\rho+\sqrt{\Delta_{R}}}{2}$, with $\eta_{1} \eta_{2}<0$ and $\eta_{1}+\eta_{2}=\rho$. In this case the eigenvalues have opposite signs. Therefore the steady state point $\left(k_{R}, q_{R}\right)$ is a saddle point.

The corresponding capital stocks, check $k_{C N 1}<k_{R}<k_{o r}<k_{C N 2}$. Since $f$ is strictly concave,

$$
\left\{\begin{array}{l}
\mu_{21}=f^{\prime}\left(k_{R}\right)-f^{\prime}\left(k_{C N 1}\right)<0<\mu_{11}=f^{\prime}\left(k_{C N 1}\right)-f^{\prime}\left(k_{o r}\right), \\
\mu_{12}=f^{\prime}\left(k_{C N 2}\right)-f^{\prime}\left(k_{o r}\right)<0<\mu_{22}=f^{\prime}\left(k_{R}\right)-f^{\prime}\left(k_{C N 2}\right) .
\end{array}\right.
$$

Therefore the steady state points $\left(k_{C N i}, q_{C N}\right)$ are saddle points. $E_{1}$ is saddle point with the vertical line as the stable manifold and horizontal line as the unstable manifold, $E_{3}$ is again a saddle point with the horizontal line as the stable manifold and the vertical line as the unstable manifold, while $E_{2}$ is saddle point with the line $q=\frac{\rho+\sqrt{\Delta_{R}}}{2}\left(k-k_{R}\right)+q_{R}$ as the stable manifold and the line $q=\frac{\rho-\sqrt{\Delta_{R}}}{2}\left(k-k_{R}\right)+q_{R}$ as the unstable manifold. In Figure 1, we plot the phase stability diagram in this case. 


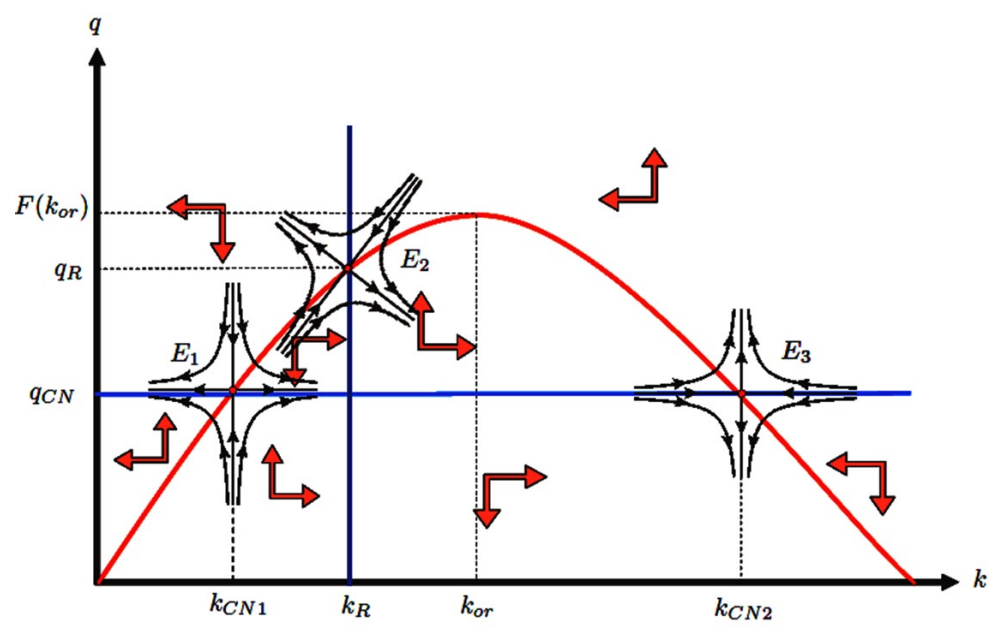

FIGURE 1. The phase stability diagram with $q_{C N}<q_{R}$

5.2. Case 2: $q_{R}=q_{C N}$. The discriminant $\Delta_{R}$ is equal to $\rho^{2}$. Then the eigenvalues $\eta_{1}$ and $\eta_{2}$ are two real with $\eta_{1}+\eta_{2}=\rho$ and $\eta_{1} \eta_{2}=\operatorname{det}\left(J_{R}\right)=h\left(q_{R}\right) f^{\prime \prime}\left(k_{R}\right)=0$. In this case one of the eigenvalues is zero and the other is equal to $\rho$. Therefore the line $q=\rho\left(k-k_{R}\right)+q_{R}$ is unstable critical line.

The corresponding capital stocks, check $k_{R}=k_{C N 1}<k_{o r}<k_{C N 2}$. Since $f$ is strictly concave,

$$
\left\{\begin{array}{l}
\mu_{21}=f^{\prime}\left(k_{R}\right)-f^{\prime}\left(k_{C N 1}\right)=0<\mu_{11}=f^{\prime}\left(k_{C N 1}\right)-f^{\prime}\left(k_{o r}\right)=\rho \\
\mu_{12}=f^{\prime}\left(k_{C N 2}\right)-f^{\prime}\left(k_{o r}\right)<0<\mu_{22}=f^{\prime}\left(k_{R}\right)-f^{\prime}\left(k_{C N 2}\right) .
\end{array}\right.
$$

Therefore the line $q=\rho\left(k-k_{C N 1}\right)+q_{C N}$ is unstable critical line, and $\left(k_{C N 2}, q_{C N}\right)$ is a saddle point.

$E_{1}$ coincides with $E_{2}$, in this case the line $q=\rho\left(k-k_{R}\right)+q_{R}$ is unstable critical line, while $E_{3}$ is a saddle point with the horizontal line as the stable manifold and the vertical line as the unstable manifold. Figure 2, a summarizes these results.

5.3. Case 3: $q_{R}<q_{C N}<F\left(k_{o r}\right)$. The corresponding capital stocks, check $k_{R}<k_{C N 1}<k_{o r}<$ $k_{C N 2}$. Since $f$ is strictly concave,

$$
\left\{\begin{array}{l}
0<\mu_{21}=f^{\prime}\left(k_{R}\right)-f^{\prime}\left(k_{C N 1}\right) \text { and } 0<\mu_{11}=f^{\prime}\left(k_{C N 1}\right)-f^{\prime}\left(k_{o r}\right) \\
\mu_{12}=f^{\prime}\left(k_{C N 2}\right)-f^{\prime}\left(k_{o r}\right)<0<\mu_{22}=f^{\prime}\left(k_{R}\right)-f^{\prime}\left(k_{C N 2}\right)
\end{array}\right.
$$

Therefore the steady state points $\left(k_{C N 1}, q_{C N}\right)$ is an unstable node and $\left(k_{C N 2}, q_{C N}\right)$ is a saddle point. 


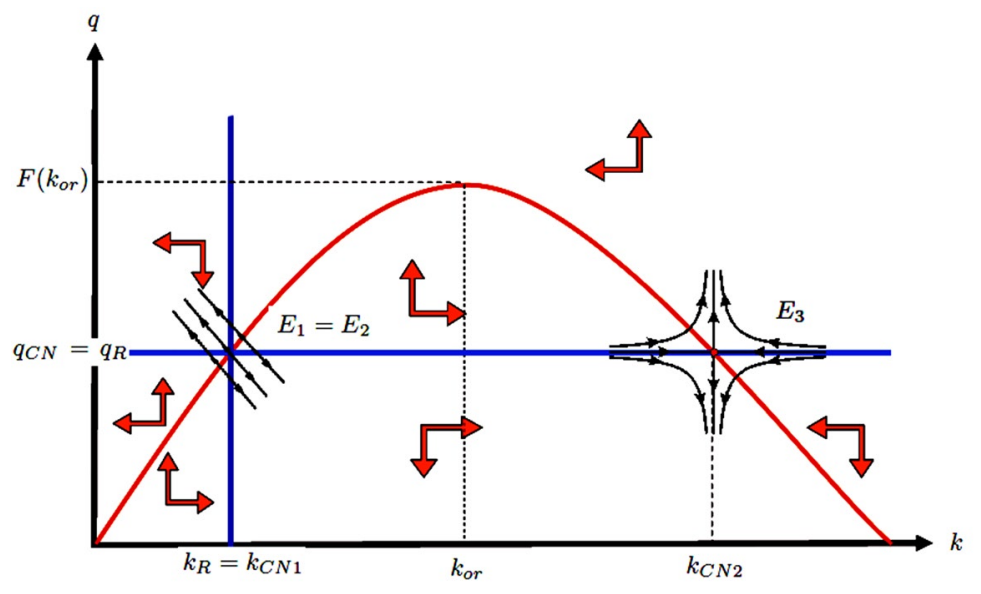

FIGURE 2. The phase stability diagram with $q_{R}=q_{C N}$.

- If $\Delta_{R}=\rho^{2}-4 h\left(q_{R}\right) f^{\prime \prime}\left(k_{R}\right)>0$, the eigenvalues $\eta_{1}$ and $\eta_{2}$ are two real, such that $\eta_{1}+\eta_{2}=\rho$ and $\eta_{1} \eta_{2}=h\left(q_{R}\right) f^{\prime \prime}\left(k_{R}\right)>0$. Therefore $\left(k_{R}, q_{R}\right)$ is an unstable node.

- If $\Delta_{R}=\rho^{2}-4 h\left(q_{R}\right) f^{\prime \prime}\left(k_{R}\right)=0$, there exist a repeated real eigenvalue equal to $\frac{\rho}{2}$ with only one eigenvector $\left(1, \frac{\rho}{2}\right)$. Therefore $\left(k_{R}, q_{R}\right)$ is an unstable improper node.

- If $\Delta_{R}=\rho^{2}-4 h\left(q_{R}\right) f^{\prime \prime}\left(k_{R}\right)<0$, we have two complex conjugate eigenvalues $\eta_{1}=\alpha+i \beta$ and $\eta_{2}=\alpha-i \beta$, with $\eta_{1}+\eta_{2}=2 \alpha=\rho>0$.Therefore $\left(k_{R}, q_{R}\right)$ is an unstable focus.

$E_{1}$ is a unstable node, and $E 3$ is a saddle point with the horizontal line as the stable manifold and the vertical line as the unstable manifold, while $E_{2}$ is an unstable node if $4 h\left(q_{R}\right) f^{\prime \prime}\left(k_{R}\right)<\rho^{2}$, an unstable improper node if $4 h\left(q_{R}\right) f^{\prime \prime}\left(k_{R}\right)=\rho^{2}$ and an unstable focus if $4 h\left(q_{R}\right) f^{\prime \prime}\left(k_{R}\right)>\rho^{2}$, as shown in figure 3. 

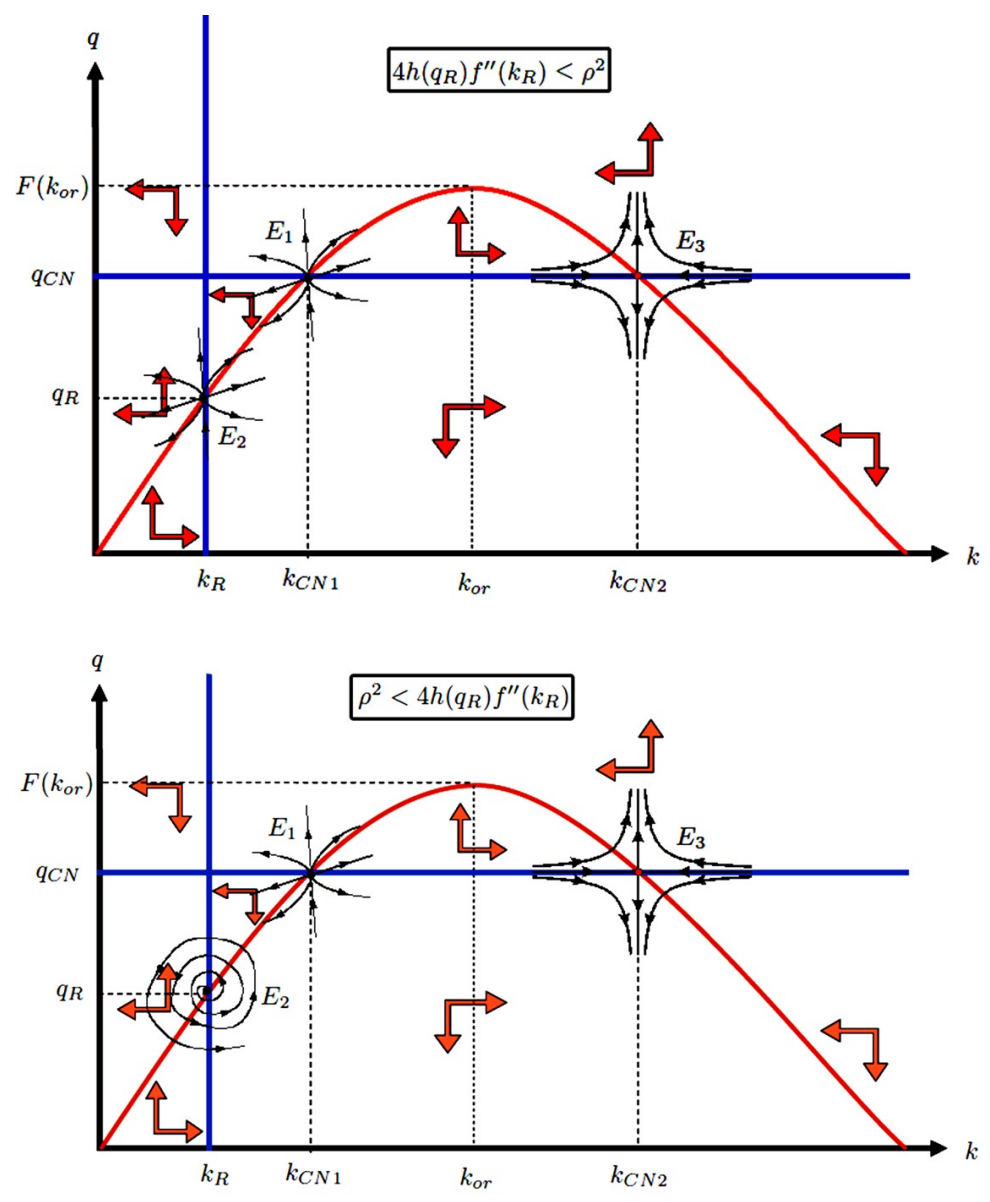

FIGURE 3. The phase stability diagram with $q_{R}<q_{C N}<F\left(k_{o r}\right)$. 
5.4. Case 4: $q_{C N}=F\left(k_{o r}\right)$. The corresponding capital stocks, check $k_{R}<k_{C N 1}=k_{o r}=k_{C N 2}$. Since $f$ is strictly concave,

$$
u_{1 i}=f^{\prime}\left(k_{C N i}\right)-f^{\prime}\left(k_{o r}\right)=0<u_{2 i}=f^{\prime}\left(k_{R}\right)-f^{\prime}\left(k_{C N i}\right)=\rho .
$$

In this case one of the eigenvalues is zero and the other is equal to $\rho$. Hence the line $q=q_{\text {or }}$ is unstable critical line.

$E_{1}$ coincides with $E_{3}$, the line $q=q_{o r}$ is unstable critical line, while $E_{2}$ is an unstable node if $4 h\left(q_{R}\right) f^{\prime \prime}\left(k_{R}\right)<\rho^{2}$, an unstable improper node if $4 h\left(q_{R}\right) f^{\prime \prime}\left(k_{R}\right)=\rho^{2}$ and an unstable focus if $4 h\left(q_{R}\right) f^{\prime \prime}\left(k_{R}\right)>\rho^{2}$, as illustrated in figure 4 .
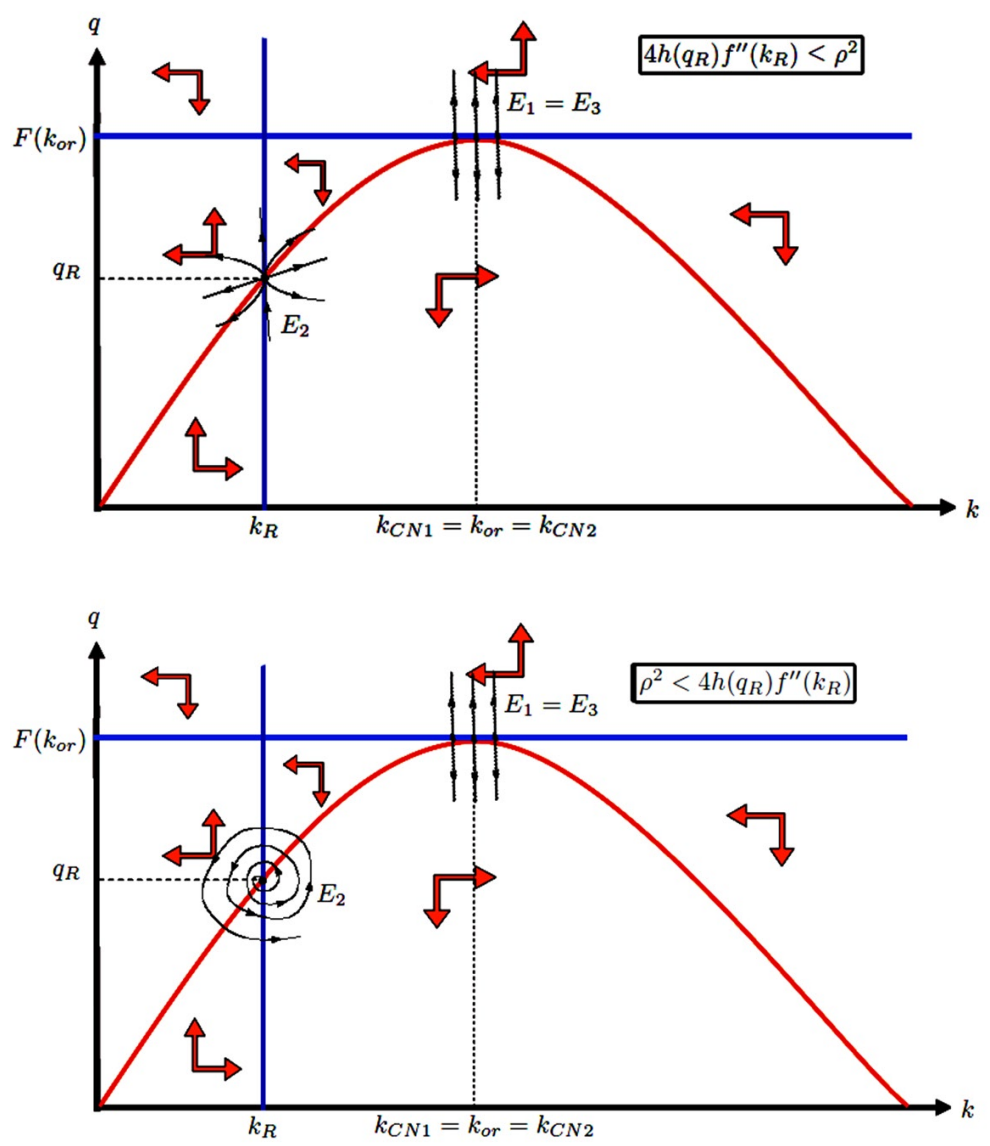

FIGURE 4. The phase stability diagram with $q_{R}<q_{C N}=F\left(k_{o r}\right)$.

5.5. Case 5: $q_{C N}>F\left(k_{o r}\right)$. Here, there exists a unique steady state point $E_{2}$, which is an unstable node if $4 h\left(q_{R}\right) f^{\prime \prime}\left(k_{R}\right)<\rho^{2}$, an unstable improper node if $4 h\left(q_{R}\right) f^{\prime \prime}\left(k_{R}\right)=\rho^{2}$ and an unstable focus if $4 h\left(q_{R}\right) f^{\prime \prime}\left(k_{R}\right)>\rho^{2}$, as in regimes case 3 and 4 . 


\section{Conclusion}

In this paper we have studied the properties of a dynamic oligopoly game with hyperbolic demand, cubic cost and Ramsey capital stock accumulation. We proved that the proposed model has an unique optimal solution and we also shown that equilibria produced by this model, Ramsey's and Nash equilibria, are stable in the saddle point sense.

\section{REFERENCES}

[1] G. Bigi, M. Passacantando, Differentiated oligopolistic markets with concave cost functions via Ky Fan inequalities, Decisions Econ. Finan. 40 (2017), 63-79.

[2] S. Lahiri, A simple model of production and trade in an oligopolistic market: back to basics, Pure Math. Appl. 27 (2018), 43-80.

[3] J.C. Cuaresma, T. Palokangas, A. Tarasyev, Dynamic Systems, Economic Growth, and the Environment. Springer, Berlin Heidelberg, 2009.

[4] Y. Tanaka, Profitability of price and quantity strategies in an oligopoly, J. Math. Econ. 35 (2001), 409-418.

[5] F.P. Ramsey, A mathematical theory of saving. Econ. J. 38 (1928), 543-559.

[6] R. Cellini, L. Lambertini, Weak and Strong Time Consistency in a Differential Oligopoly Game with Capital Accumulation. J. Optim. Theory Appl. 138 (2008), 17-26.

[7] L. Lambertini, Oligopoly with Hyperbolic Demand: A Differential Game Approach, J. Optim. Theory Appl. 145 (2010), 108-119.

[8] L. Lambertini, A. Palestini, On the feedback solutions of differential oligopoly games with hyperbolic demand curve and capacity accumulation, Eur. J. Oper. Res. 236 (2014), 272-281.

[9] S. Erfle, Deriving a cubic total cost function from a cubic total cost curve, Int. J. Econ. Commerce Manage. 2 (2014), 1-12.

[10] W. Diewert, Duality Approaches to Microeconomic Theory, in Kenneth Arrow and Michael Intriligator (ed.), Handbook of Mathematical Economics, vol II, North-Holland Publishing Company, Amesterdam, 535-599, 1982.

[11] W. Baumol, J. Panzar and R. Willig, Contestable Markets and the Theory of Industry Structure, Revised Edition, Cambridge, Harcout Brace Jovanovich, Publishers, New York, 1988.

[12] R. Cellini, L. Lambertini, A differential oligopoly game with differentiated goods, European Journal of Operational Research 176 (2007) 1131-1144 and sticky prices.

[13] M. Greer, Electricity Cost Modeling Calculations, Butterworth-Heinemann, Boston, 2012.

[14] O. Nikutowski, V. Leis, R.K.Frhr. von Weizsacker, Cubic Cost Functions and Major Market Structures, J. Econ. Educ. 44 (2013) 91?91.

[15] R. Martins, A. Fortunato, F. Coelho, Cost Structure of the Portuguese Water Industry: a Cubic Cost Function Application, GEMF Working Papers 2006-09, GEMF, Faculty of Economics, University of Coimbra.

[16] J.W. Friedman, Oligopoly and the Theory of Games, Amsterdam, North-Holland, 1977.

[17] M. Yildizoglu, Introduction a la theorie des jeux, Dunod, Paris, (2003). 
[18] J. Tirole, The Theory of Industrial Organization, The MIT Press, Cambridge, Massachusetts, 1988. 